\title{
Intracellular Regulation of Evodiamine-Induced A375-S2 Cell Death
}

\author{
Ying Zhang, ${ }^{a}$ Li-Jun Wu ${ }^{b}$ Shin-ichi Tashiro, ${ }^{c}$ Satoshi Onodera, ${ }^{c}$ and Takashi IkeJima ${ }^{*, a}$ \\ ${ }^{a}$ China-Japan Research Institute of Medical and Pharmaceutical Sciences, Shenyang Pharmaceutical University; \\ ${ }^{b}$ Department of Phytochemistry, Shenyang Pharmaceutical University; Shenyang 110016, China: and ${ }^{c}$ Department of \\ Clinical and Biomedical Sciences, Showa Pharmaceutical University; Tokyo 194-8543, Japan. \\ Received April 25, 2003; accepted July 16, 2003
}

\begin{abstract}
We have reported that in A375-S2 cells, evodiamine isolated from Evodia rutaecarpa induces cell death of human melanoma, A375-S2, through two distinct pathways: apoptosis and necrosis. In the present study, we further demonstrate two different mechanisms by which evodiamine induces apoptosis and necrosis. Although caspase-1 and -10 inhibitors failed to block cell death, pan-caspase inhibitor and caspase-3, -8, and -9 inhibitors had marked inhibitory effects on apoptosis induced by $15 \mu_{\mathrm{M}}$ evodiamine. Furthermore, evodiamine-induced activation of caspase-3 resulted in the down-regulation of anti-apoptotic Bcl-2 expression and up-regulation of proapoptotic Bax expression. After $24 \mathrm{~h}$ incubation with evodiamine, no caspase inhibitor had any influence on cell death, but p38 mitogen-activated protein kinase (MAPK) inhibitor (SB203580) attenuated cell death; in contrast, extracellular signal-regulated protein kinase (ERK) MAPK inhibitor (PD98059) augmented cell death, as was further confirmed by cotreatment with SB203580 or PD98059 and pan-caspase inhibitor. Moreover, evodiamine increased the phosphorylation of $\mathrm{p38}$ and decreased the expression and phosphorylation of ERK in caspase-independent necrosis. Consequently, evodiamine induced the caspase- and Bax-mediated apoptosis at an early stage, but, initiated MAPKs-dependent necrosis at a later stage.
\end{abstract}

Key words evodiamine; human melanoma cell; apoptosis; necrosis

Evodiamine isolated from Evodia rutaecarpa Bentham (go-shu-yu in Japanese) of fruits has been reported to increase arterial pressure in vivo, and to possess anti-tumor, anti-nociceptive, vasorelaxant ${ }^{1)}$ and catecholamine-seretory properties. Ogasawara et al. reported that evodiamine had a marked inhibitory influence on tumor cell migration in vitro. ${ }^{2)}$ In our previous studies, evodiamine induced cell death in the cell lines A375-S2, ${ }^{3} \mathrm{HeLa}^{3,4)}$ and L929 cells, ${ }^{5}$ but had less effect on human peripheral blood mononuclear cells. ${ }^{5)}$ Our previous studies on A375-S2 cells treated with evodiamine showed that in these cells, apoptotic and necrotic pathways were initiated before and after $24 \mathrm{~h}$, respectively. ${ }^{3)}$ In this study, we demonstrate that the caspase family, the mitochondrial Bcl-2 family and the mitogen-activated protein kinase (MAPK) family play important roles in evodiamineinduced A375-S2 cell death at different culture stages.

Caspases are cysteine proteases that mediate apoptosis by proteolysis of specific substrates including anti-apoptotic mitochondrial protein $\mathrm{Bcl}-2{ }^{6}{ }^{6}$ The $\mathrm{Bcl}-2$ family functions to either promote or inhibit apoptosis. ${ }^{7)}$ Once apoptosis was initiated, Bcl-2 was cleaved by caspase- 3 to attenuate its antiapoptotic effects. ${ }^{8)}$ The subtle balance of the Bcl-2/Bax complex might modulate the anti- or pro- apoptotic effect. The overexpression of proapoptotic Bax induced the loss of the mitochondrial membrane potential, resulting in the release of cytochrome c. Subsequently, this release presumably led to the activation of caspase-9, which then activated downstream effector caspases including caspase-3, finally culminating in apoptosis. ${ }^{9)}$

The MAPKs family also plays an important role in cell growth and death. Extracellular signal-regulated protein kinase (ERKs) are activated in response to growth factor stim$\mathrm{uli}^{10)}$ resulting in protection against apoptosis and necrosis, ${ }^{11)}$ whereas the stimulation of c-Jun N-terminal kinase (JNK) and p38 signaling pathways contributes to cell death including apoptosis ${ }^{12)}$ and necrosis. ${ }^{13,14)}$
In the present study, we demonstrated that down-regulation of Bcl-2 and up-regulation of Bax amplified the activation of caspase cascade, resulting in apoptosis in A375-S2 cells. In addition, simultaneous activation of $\mathrm{p} 38$ MAPK and the inhibition of ERK activity contributed to evodiamine-induced necrosis at later stages.

\section{MATERIALS AND METHODS}

Reagents Evodiamine was purchased from the Beijing Institute of Biological Products (Beijing, China); Its purity was measured by HPLC and determined to be about $98 \%$. Evodiamine was dissolved in dimethyl sulfoxide $\left(\mathrm{Me}_{2} \mathrm{SO}\right)$ to make a stock solution. $\mathrm{Me}_{2} \mathrm{SO}$ concentration was kept below $0.001 \%$ in all the cell cultures and did not exert any detectable cytotoxicity.

Pan-caspase inhibitor (z-VAD-fmk), caspase-3 (z-DEVDfmk), capase-8 (z-IETD-fmk), caspase-9 (z-LEHD-fmk), and caspase-10 (z-AEVD-fmk) inhibitors were purchased from Enzyme Systems (CA, U.S.A.), and caspase-1 inhibitor (AcYVAD-cmk) was obtained from Bachem (Bubendorf, Swizerland). Poly (ADP-ribose) polymerase (PARP) inhibitor (DPQ), p38 MAPK inhibitor (SB203580), JNK inhibitor (SP600125), and ERK inhibitor (PD98059) were purchased from Calbiochem (CA, U.S.A.). Rabbit polyclonal antibodies against p38, ERK2, phospho-ERK, and horseradish peroxidase-conjugated secondary antibody (goat-anti-rabbit) were purchased from Santa Cruz Biotechnology (Santa Cruz, CA, U.S.A.). Rabbit polyclonal anti-Bcl-2 and anti-Bax were purchased from Oncogene Research Products (MA, U.S.A.). Rabbit polyclonal anti-phospho-p38 was purchased from Techne (CA, U.S.A.). Caspase-3 and -8 apoptosis kits were from Santa Cruz Biotechnology, and a caspase-9 colorimetric activity assay kit was purchased from Chemicon International (CA, U.S.A.).

Cell Culture Human malignant melanoma A375-S2 
cells were obtained from the American Type Culture Collection (ATCC, \#CRL 1872, U.S.A.). The cells were cultured in RPMI-1640 medium (GIBCO, U.S.A.) supplemented with $5 \%$ fetal bovine serum (FBS) (Dalian Biological Reagent Factory, Dalian, China) and $0.03 \%$ L-glutamine at $37^{\circ} \mathrm{C}$ in $5 \% \mathrm{CO}_{2}$. Cells in the exponential phase of growth were used in the experiments.

Cytotoxicity Assay A375-S2 cells were incubated at $5 \times 10^{3}$ cells/well in 96-well plates (NUNC, Denmark). The cells were incubated with z-VAD-fmk, Ac-YVAD-cmk, zDEVD-fmk， z-IETD-fmk， z-LEHD-fmk， z-AEVD-fmk, SB203580, SP600125, or PD98059 at given concentrations for $1 \mathrm{~h}$, then treated with evodiamine for different time periods. Cell growth was measured with a plate reader (Bio-Rad, U.S.A.) by 3-(4,5-dimethylthiazol-2-yl)-2,5-diphenyltetrazolium (MTT) assay at different time points as previously described. The percentage of cell growth inhibition was calculated as follows:

cell death $(\%)=\left[A_{570}(\right.$ control $)-A_{570}($ evodiamine $\left.)\right] / A_{570}($ control $) \times 100$

DNA Fragmentation Assay ${ }^{3)}$ A375-S2 cells $\left(1 \times 10^{6}\right.$ cells) were seeded and treated with $15 \mu \mathrm{m}$ evodiamine. Both floating and adherent cells were scraped off and collected in medium, washed three times with phosphate-buffered saline, and resuspended in lysis buffer $(10 \mathrm{~mm}$ Tris- $\mathrm{HCl}, \mathrm{pH} 7.4$, 10 mм EDTA, pH 8, and 0.5\% Triton X-100). After a 30-min incubation on ice, the lysate was incubated with proteinase $\mathrm{K}$ $40 \mu \mathrm{g} / 1$ (Merck, U.S.A.) at $37^{\circ} \mathrm{C}$ for $2 \mathrm{~h}$, then extracted with $0.5 \% 5 \mathrm{M} \mathrm{NaCl}$ and $50 \%$ 2-propanol, incubated overnight at $-20^{\circ} \mathrm{C}$, and centrifuged at $7000 \times \boldsymbol{g}$ for $20 \mathrm{~min}$. The supernatant was washed with $70 \%$ ethanol and centrifuged and pellets were dried and suspended in $10 \mathrm{~mm}$ Tris- $\mathrm{HCl}(\mathrm{pH}$ 7.4) and 1 mm EDTA. DNA was incubated with RNase A $40 \mu \mathrm{g} / 1$ (Sigma, U.S.A.) at $37^{\circ} \mathrm{C}$ for $60 \mathrm{~min}$, separated by $2 \%$ agarose gel electrophoresis and stained with ethidium bromide $0.1 \mathrm{mg} / \mathrm{l}$.

Caspase Activity Assay A375-S2 cells were cultured at $1 \times 10^{6}$ cells and treated with or without evodiamine $15 \mu \mathrm{M}$ for different time periods. The enzymatic reaction for caspase- 3 and -8 activity was carried out with the caspase- 3 substrate (DEVD-AFC) and caspase-8 substrate (IETD-AFC), according to the manufacturer's instruction. The activity of caspase- 9 was measured by caspase- 9 colorimetric activity assay kit.

Western Blot Analysis Cells were left untreated or treated with evodiamine, then harvested, washed in PBS, and whole cell extracts were prepared under reducing condition. ${ }^{8,9)}$ Equivalent amounts of protein were separated by SDS-PAGE and wet-electrotransferred onto nitrocellulose membrane. Proteins were detected with antibodies against Bcl-2, Bax, p38, phospho-p38, ERK, and phospho-ERK, followed by addition of horseradish peroxidase-conjugated secondary antibody.

Statistical Analysis All results were confirmed in at least three separate experiments. $\mathrm{IC}_{50}$ values were calculated by regression analysis of the concentration response data. Data of the representatives were analyzed for statical significance by Student's $t$-test. $p$-Values of less than 0.05 were considered significant.

\section{RESULTS}

Effects of Caspases on Evodiamine-Induced Cytotoxicity in A375-S2 Cells Fifteen $\mu \mathrm{m}$ evodiamine induced about $50 \%$ A375-S2 cell death at $24 \mathrm{~h}$ in a dose-dependent manner (Fig. 1). At the early stage before $24 \mathrm{~h}$ incubation with $15 \mu \mathrm{M}$ evodiamine, caspase-1 inhibitor (Ac-YVAD-cmk) and caspase-10 inhibitor (z-AEVD-fmk) failed to block apoptosis. However, the inhibitors of pan-caspase, caspase-3, -8 , and -9 (z-VAD-fmk, z-DEVD-fmk, z-IETD-fmk and z-LEDH-fmk, respectively) had inhibitory effects on cell death (Fig. 2). In particular, the effects of caspase- 3 and caspase-9 inhibitors were more significant than others. After $24 \mathrm{~h}$, none of the caspase inhibitors affected A375-S2 cell apoptosis, as we previously reported. ${ }^{3)}$ These findings were confirmed by the measurement of caspase-3, -8 , and -9 activities. Twelve hours after treatment with evodiamine, activity of upstream caspase- 8 reached a peak, 2.5 times higher than those of the negative control group. Simultaneously, caspase-9 activity was also increased to 3.5 times of control, which further augmented the delayed caspase- 3 activation, reaching a four-fold increase of the control level at $24 \mathrm{~h}$ (Fig. 3).

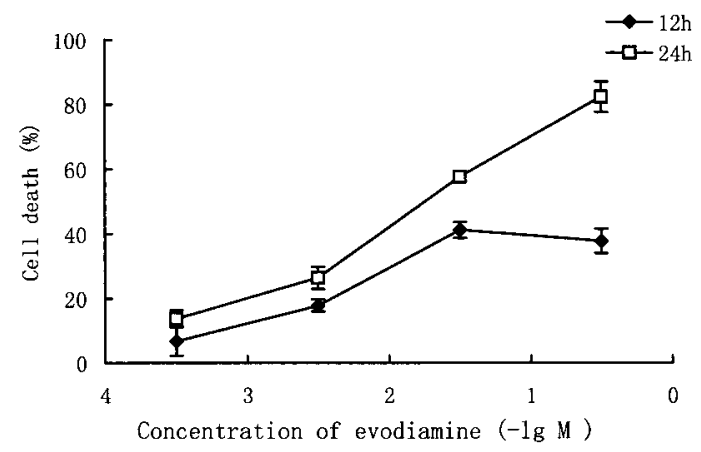

Fig. 1. Inhibitory Effect of Evodiamine on A375-S2 Cell Growth

Cells $\left(5 \times 10^{3} /\right.$ well $)$ were treated with evodiamine for 12 and $24 \mathrm{~h}$, and the percentage of cell death was determined by MTT assay $\left(\mathrm{M}=3 \times 10^{-4}, 3 \times 10^{-3}, 3 \times 10^{-2}\right.$, $\left.3 \times 10^{-1} \mathrm{mmol} \cdot 1^{-1}\right) . n=3$. Mean \pm S.D.

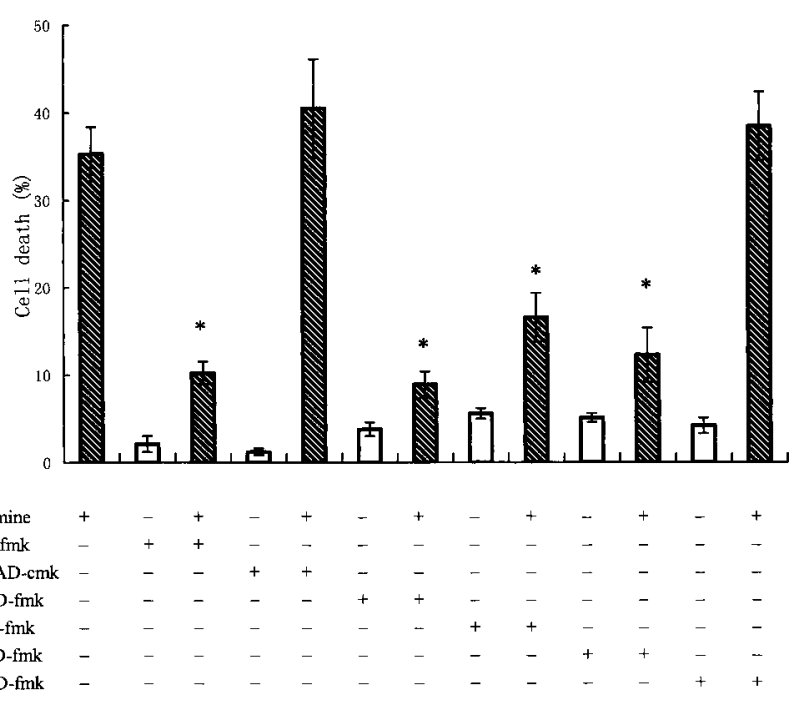

Fig. 2. Effects of Caspase Inhibitors on Evodiamine-Induced Cell Death

A375-S2 cells were treated with $15 \mu \mathrm{M}$ evodiamine for $12 \mathrm{~h} .20 \mu \mathrm{M}$ Pan-caspase inhibitor (z-VAD-fmk), caspase-1 inhibitor (Ac-YVAD-cmk), caspase-3 inhibitor (zDEVD-fmk), caspase-8 inhibitor (z-IETD-fmk), caspase-9 inhibitor (z-LEHD-fmk), or caspase-10 inhibitor ( $\mathrm{z}$-AEVD-fmk) was added $1 \mathrm{~h}$ prior to the treatment with evodiamine. $n=3$. Mean \pm S.D. $* p<0.05 v s$. evodiamine alone group. 


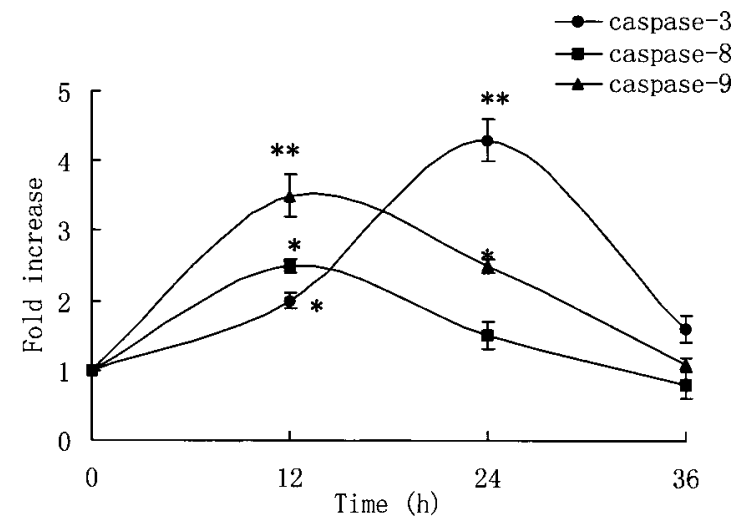

Fig. 3. Effects of Evodiamine on the Activation of Caspase- $3,-8$ and -9 in A375-S2 Cells

Cells were treated with $15 \mu \mathrm{M}$ evodiamine for 12,24 , and $36 \mathrm{~h} . n=3$. Mean \pm S.D. $* p<0.05, * * p<0.001 v s$. without evodiamine group.

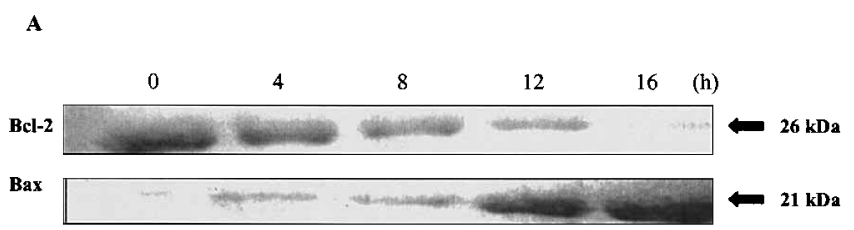

B

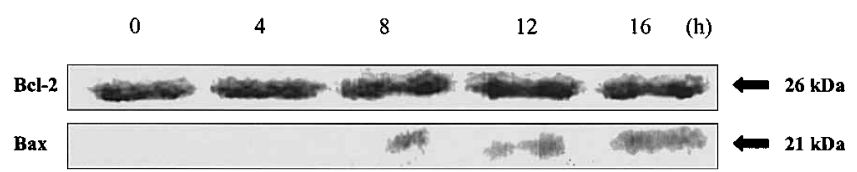

Fig. 4. Effects of Evodiamine on Bcl-2 and Bax Expression in the Absence or Presence of Caspase-3 Inhibitor (z-DEVD-fmk)

A375-S2 cells were treated with $15 \mu \mathrm{M}$ evodiamine for the indicated times (A, B), and $20 \mu \mathrm{m}$ z-DEVD-fmk was added $1 \mathrm{~h}$ prior to the administration of evodiamine (B). Expression of Bcl-2 and Bax was measured by Western immunoblot analysis.

The Involvement of Bcl-2 Family Protein in Evodiamine-Induced A375-S2 Cell Apoptosis With 4 to $16 \mathrm{~h}$ incubation with evodiamine, expression of mitochondrial anti-apoptotic protein $\mathrm{Bcl}-2$ was down-regulated, while the level of proapoptotic protein, Bax, was up-regulated (Fig. 4A). These results were reversed by caspase- 3 inhibitor (Fig. $4 \mathrm{~B}$ ), suggesting that the activation of caspase- 3 was required for the change of Bcl-2 and Bax balance by evodiamine.

Regulations of MAPKs in the Caspase and PARP-Independent Necrosis Initiated by Evodiamine at Later Culture Stage We have demonstrated that evodiamine induced necrosis in a caspase-independent manner after $24 \mathrm{~h}$ drug treatment. ${ }^{3)}$ It was reported that some necrotic cases were thought to be caspase-independent and PARP-mediated. ${ }^{15)}$ Thus, we examined the possibility that PARP participated in evodiamine-induced necrosis. Although an unusually high dose $(150 \mu \mathrm{M})$ of DPQ increased evodiamine-induced cell death from 66.7 to $87 \%$ at $36 \mathrm{~h}, 150 \mu \mathrm{M}$ DPQ alone induced about $40 \%$ cell death in the absence of evodiamine; neither 6 nor $30 \mu \mathrm{M}$ DPQ influenced cell death. These findings showed that the PARP specific inhibitor (DPQ) failed to block cell death, suggesting that evodiamine-induced necrosis was PARP- and caspase-independent (Fig. 5).

The specific inhibitors for p38 (SB203580), JNK
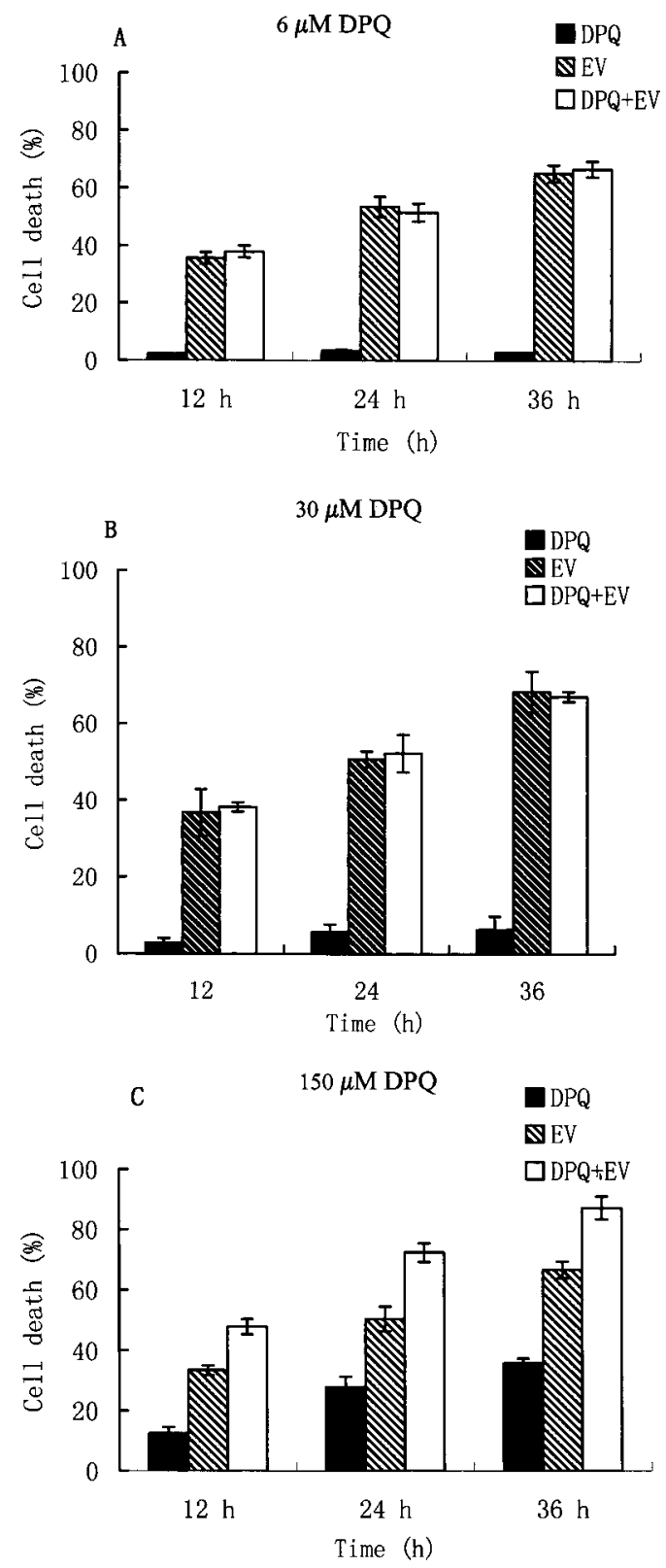

Fig. 5. The Specific PARP Inhibitor DPQ Had No Influence on Cell Death Induced by Evodiamine

A375-S2 cells were cultured in the presence of $6 \mu \mathrm{M}$ (A), $30 \mu \mathrm{M}$ (B), and $150 \mu \mathrm{M}$ DPQ (C), $1 \mathrm{~h}$ prior to the treatment with $15 \mu \mathrm{M}$ evodiamine (EV), then incubated for 12 , 24 and 36 h. $n=3$. Mean \pm S.D.

(SP600125), and ERK (PD98059) were applied to assess the function of MAPKs to evodiamine-induced A375-S2 cell death. As previously reported, no MAPK inhibitor had any effect until $12 \mathrm{~h}$ incubation with evodiamine when the major cause of cell death was apoptosis. ${ }^{3)}$ However, after $24 \mathrm{~h}$ incubation with evodiamine, $20 \mu \mathrm{M}$ SB203580 partially blocked necrosis. On the contrary, when ERK signaling was inhibited by $20 \mu \mathrm{M}$ PD98059, the cytotoxic effect of evodiamine on A375-S2 cells was augmented, whereas PD98059 alone had a minimum effect. JNK inhibitor did not affect the evodiamine-induced death of A375-S2 cells (Fig. 6).

Pan-caspase inhibitor had an inhibitory effect on evodiamine-induced apoptosis at $12 \mathrm{~h}$, however, this effect disappeared after $24 \mathrm{~h}$. Cotreatment with SB203580 and z-VADfmk markedly blocked evodiamine-induced cell death over 


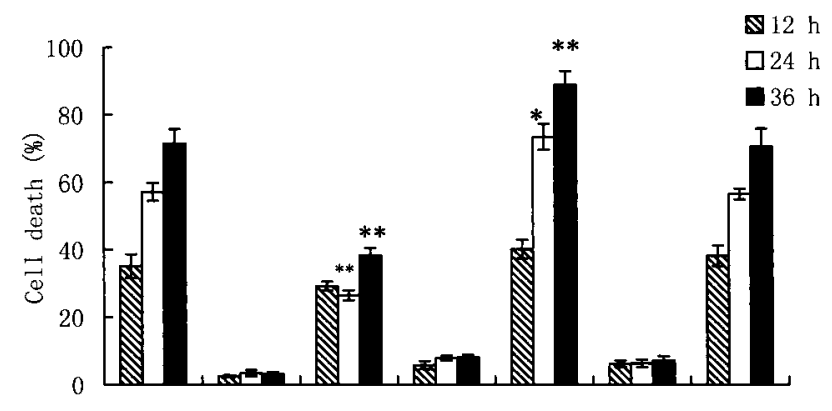

$\begin{array}{llllllll}\text { Evodiamine } & + & - & + & - & + & - & + \\ \text { SB203580 } & - & + & + & - & - & - & - \\ \text { PD98059 } & - & - & - & + & + & - & - \\ \text { SP600125 } & - & - & - & - & - & + & +\end{array}$

Fig. 6. Opposite Effects of MAPK Inhibitors on Necrosis Induced by Evodiamine

A375-S2 cells were treated with $15 \mu \mathrm{M}$ evodiamine for $24 \mathrm{~h} .10 \mu \mathrm{M}$ p38 inhibitor (SB203580), $10 \mu \mathrm{M}$ ERK inhibitor (PD98059) or $20 \mu \mathrm{M}$ JNK inhibitor (SP600125) were added $1 \mathrm{~h}$, prior to the treatment with evodiamine. $n=3$. Mean \pm S.D. $* p<0.05$, $* * p<0.001 v s$. evodiamine alone group.

$12 \mathrm{~h}$ (Fig. 7A), and simultaneously inhibited a smear-like degradation of DNA at $24 \mathrm{~h}$ (Fig. 7B). In contrast, PD98059 attenuated the protectional effects of pan-caspase inhibitor against cell death, and also enhanced the necrotic DNA degradation (Fig. 7B). JNK inhibitor failed to influence the function of z-VAD-fmk (Fig. 7A). These results indicated that activated p38 MAPK contributed to evodiamine-induced necrosis.

To further confirm this, the effects of evodiamine on the p38 and ERK phosphorylation were examined. Evodiamine did not alter p38 protein expression, but increased the phosphorylation of p38 (Fig. 8). Unlike p38 MAPK, both the protein expression and phosphorylation of ERK were markedly down-regulated by evodiamine. These results suggested that ERK and p38 had opposite effects on evodiamine-induced necrosis.

\section{DISCUSSION}

Depending on cell type and stimuli, cells die through one of two distinct ways: apoptosis or necrosis. Biochemical and morphological characteristics of these death ways are significantly different, and can be initiated by one or more of various stimuli (e.g., cytokines, ischemia, heat, irradiation and pathogens). Signaling pathways, such as death receptors (e.g., Fas, TNF receptor), kinase cascades, and mitochondria, participate in both processes, and by modulating these pathways it is possible to switch from apoptosis to necrosis.

Our previous studies demonstrated that evodiamine initiated apoptosis in A375-S2 cells before 24h. ${ }^{3)}$ Thus, in the present study, we showed that activation of caspase-3, -8 , and -9 contributed to apoptosis in response to evodiamine administration. In addition, A375-S2 cells treated with evodiamine exhibited elevated levels of proapoptotic Bax expression, while anti-apoptotic Bcl-2 was down regulated. It was reported that Bcl-2 was cleaved by caspase- 3 , thus causing a pro-apoptotic function similar to that of Bax. ${ }^{8}$ Furthermore, the up-regulation of Bax was believed to induce cytochrome c efflux, which led to the activation of caspase-9; subsequently, downstream caspase-3 was activated. ${ }^{9)}$ In our studies, when the activation of caspase- 3 was increased to about
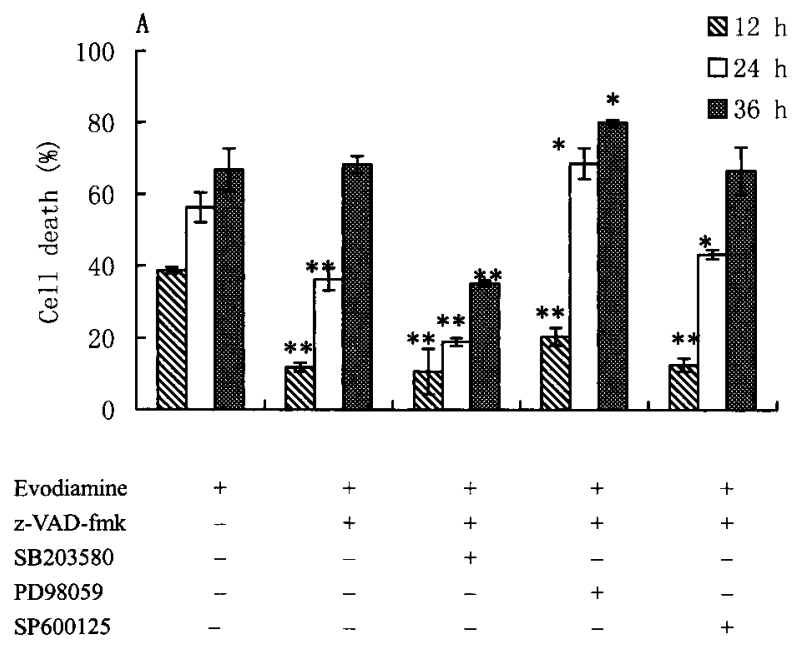

B

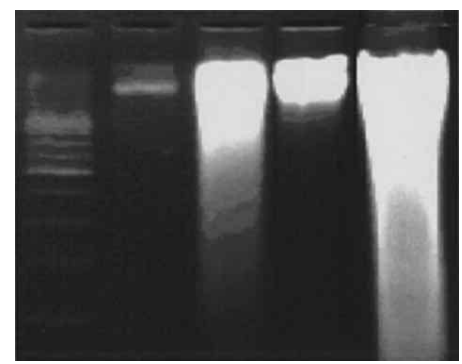

\begin{tabular}{lllll}
\multicolumn{1}{r}{ M } & & & & \\
Evodiamine & - & + & + & + \\
z-VAD-fmk & - & - & + & + \\
SB203580 & - & - & + & - \\
PD98059 & - & - & - & +
\end{tabular}

Fig. 7. The Effects of the Pan-Caspase Inhibitor (z-VAD-fmk) and MAPK Inhibitors on Evodiamine-Induced Cell Death

A375-S2 cells were treated with $20 \mu \mathrm{m}$ z-VAD-fmk and MAPK inhibitors for $1 \mathrm{~h}$, including $10 \mu \mathrm{M}$ p38 inhibitor (SB203580), $10 \mu \mathrm{M}$ ERK inhibitor (PD98059), and $20 \mu \mathrm{M}$ JNK inhibitor (SP600125), prior to the administration of $15 \mu \mathrm{m}$ evodiamine, then cultured for 12,24 , and $36 \mathrm{~h}$ (A). The effects of z-VAD-fmk and $10 \mu \mathrm{M} \mathrm{SB} 203580$ or $10 \mu \mathrm{M}$ PD98059 on evodiamine-induced DNA degradation. Cells were treated with evodiamine for $24 \mathrm{~h}$ and DNA degradation was examined by $2 \%$ agarose gel electroporesis (B). M: molecular weight marker. $n=3$. Mean \pm S.D. $* p<0.05$, $* * p<0.001 v$ s. evodiamine alone group.

twice that of control at $12 \mathrm{~h}, \mathrm{Bcl}-2$ expression began to be down-regulated. Following the increase of caspase- 3 activity, Bcl-2 expression was not detected, however, Bax expression was obviously up-regulated. Furthermore, caspase-3 inhibitor not only blocked the activation of caspase-3, but also reversed the regulation of Bcl-2 and Bax by evodiamine. We also cannot dispel the possibility that evodiamine directly increased the Bcl-2 activity and decreased the Bax activity, leading to increase in caspase-3 activity. These results appear to suggest that caspase-3-dependent cleavage of Bcl-2 further activated caspase cascades as part of a positive feedback loop for executing the cells.

PARP, at the downstream of caspase-3, is a nuclear enzyme that responds to DNA damage and facilitates DNA repair. ${ }^{16)}$ Although some research has reported that the cause of PARP-dependent cell death is thought to be necrosis, which is caspase-independent, ${ }^{15}$ ) PARP did not participate in caspase-independent necrotic stages of evodiamine-induced 

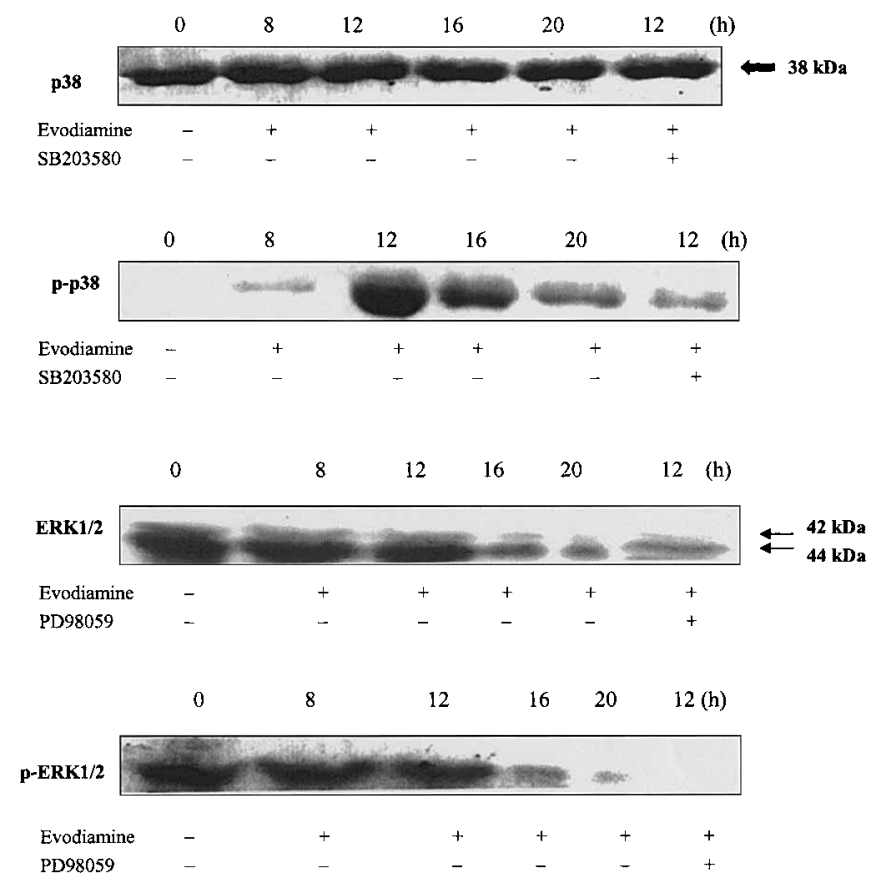

Fig. 8. Western Blot Analysis of p38, Phospho-p38, ERK and PhosphoERK

A375-S2 cells were incubated in the medium alone or the medium containing $10 \mu \mathrm{M}$ p38 inhibitor (SB203580) or ERK inhibitor (PD98059) for $1 \mathrm{~h}$, followed by $15 \mu \mathrm{M}$ evodiamine for $12 \mathrm{~h}$, or containing $15 \mu \mathrm{M}$ evodiamine in the absence of SB203580 or PD98059 for $8,12,16$, and $20 \mathrm{~h}$.

\section{A375-S2 cells.}

The members of MAPKs including ERKs, JNKs and p38 MAPK, were reported to play important roles in apoptosis and necrosis. ${ }^{12)}$ p38 and JNK MAPK signaling pathways have been thought to be related to cellular death pathways. For instance, ceramide-induced necrosis of prostate carcinoma ${ }^{13)}$ and heat shock-induced necrosis of osteoblasts are correlated with JNK activation. ${ }^{17)}$ Suppression of p38 reduced necrotic zone formation in the myocardium. ${ }^{14)}$ In our studies, p38 was activated and the phosphorylation of p38 was increased in A375-S2 cells treated with evodiamine. Furthermore, SB203580 attenuated the smear-like DNA degradation, which was a hallmark of necrosis, indicating that the activation of p38 was responsible for necrosis induced by evodiamine at later stage. We previously demonstrated that the number of necrotic cells was more than that of apoptotic cells at $36 \mathrm{~h},{ }^{3)}$ and thus concluded that evodiamine-induced necrosis was mainly due to the activation of p38 MAPK. These results were coincident with other reports that the activation of p38 MAPK was involved in the necrosis. For instance, suppression of p38 reduced necrotic zone formation in the myocardium ${ }^{18)}$ and hippocampal $\mathrm{Cal}$ region after ischemia. ${ }^{14)}$

On the contrary, activation of the ERK signaling pathway protects cells from a variety of cellular stresses. ${ }^{12)}$ ERK inhibitor augmented evodiamine-induced necrosis in A375-S2 cells. Moreover, the expression and phosphorylation of ERKs were down regulated by evodiamine. All these results were confirmed by the combination of pan-caspase inhibitor (zVAD-fmk) and SB203580 or PD98059 to test the effects on evodiamine-induced cell death.

Although the consequences of necrotic and apoptotic cell death are quite different for a whole organism, there are many examples of both models of cell death being found in the same cell. These suggest that some crossing points exist in both signal pathways. The caspase family is believed to play a key role in apoptosis, however, it may also play a signaling role in activation of other proteases such as cathepsine, calpain, and serine proteases whose involvement in necrotic execution was demonstrated in some circumstances. In our studies, caspases were demonstrated to be involved in the early apoptotic stage, but it was unclear whether caspases activated other proteases related to necrosis. In addition, we speculate that the DNA damage induced by caspase activation might be responsible for the activation of MAPKs, which initiates the expression of some pronecrotic genes. Thus, evodiamine-induced caspase-dependent apoptosis might be switched to necrosis regulated by MAPKs.

In summary, we demonstrated that evodiamine initiated two distinct intracellular regulation systems to induce in A375-S2 cell death: (1) At the early stage, evodiamine led to the activation of caspase- $3,-8$, and -9 , accompanied by the down-regulation of Bcl-2 and up-regulation of Bax, shifted to the apoptotic direction. (2) Opposite effects of ERK and p38 MAPK contributed to necrosis in response to evodiamine at later stages.

\section{REFERENCES}

1) Chiou W. F., Chou C. J., Shum A. Y., Chen C. E., Eur. J. Pharmacol., 215, 277-283 (1992).

2) Ogasawara M., Matsubara T., Suzuki H., Biol. Pharm. Bull., 24, 917920 (2001).

3) Zhang Y., Wu L. J., Tashiro S., Onodera S., Ikejima T., Acta Pharmacol. Sin., 38, (2003), (in press).

4) Fei X. F., Wang B. X., Li T. J., Tashiro S., Minami M., Xing D. J., Ikejima T., Cancer Sci., 94, 92-98 (2003).

5) Zhang Y., Zhang Q. H., Wu L. J., Tashiro S., Onodera S., Ikejima T., J. Asian Nat. Prod. Res., (2003) (in press).

6) Villa P., Kaufmann S. H., Earnshaw W. C., Trend Immunol. Biochem. Sci., 22, 386-393 (1997).

7) Korsmeryer S. J., Blood, 80, 879-886 (1992).

8) Kirsch D. G., Doseff A., Chau B. N., Lim D. S., de Souza-Pinto N. C., Hansford R., Kastan M. B., Lazebnik Y. A., Hardwick J. M., J. Biol. Chem., 274, 21155-21161 (1999).

9) Han Z. Y., Pantazis P., Wyche J. H., Kouttab N., Kidd V., J. Biol. Chem., 276, 38748-38754 (2001).

10) Davis R. J., J. Biol. Chem., 268, 14553-14556 (1993).

11) Punn A., Mockridge J. W., Farooqui S., Marber M. S., Heads R. J., Biochem. J., 350, 330-335 (2000).

12) Xia Z. G., Dikens M., Raingeaud J., Davis R. J., Greenberg M. E., Science, 270, 1336-1331 (1995).

13) Engedal N., Saatcioglu F., Prostate, 46, 289-297 (2001).

14) Sugino T., Nozaki K., Takagi Y., Hattori I., Hashimoto N., Moriguchi T., Nishida E., J. Neurosci., 20, 4506-4514 (2000).

15) Yu S. W., Wang H. M., Poitras M. F., Coombs C., Bowers W., Federoff H. J., Poirier G. G., Dawson T. M., Dawson V. L., Science, 297, 259263 (2002).

16) Mandal M., Maggirwar S. B., Sharma N., Kaufmann S. H., Sun S. C., Kumar R., J. Biol. Chem., 271, 30354 -30359 (1996).

17) Li S., Chien S., Branemark P. I., J. Orthop. Res., 17, 891-899 (1999).

18) Ma X. L., Kumar S., Gao F., Louden C. S., Lopez B. L., Christopher T. A., Wang C., Lee J. C., Feuerstein G. Z., Yue T. L., Circulation, 99, 1685-1691 (1999). 